REVISTA CIENCIAS BIOMÉDICAS

ARTÍCULOS ORIGINALES

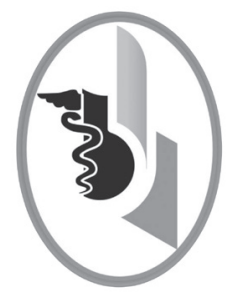

\title{
CARACTERIZACIÓN DE LOS PACIENTES CON TRATAMIENTO ENDOVASCULAR DE ANEURISMAS DISECANTES DE CIRCULACIÓN CEREBRAL POSTERIOR EN CARTAGENA DE INDIAS
}

\author{
CHARACTERIZATION OF PATIENTS WITH ENDOVASCULAR \\ TREATMENT OF DISSECTING ANEURYSMS OF POSTERIOR \\ CEREBRAL CIRCULATION IN CARTAGENA DE INDIAS
}

\author{
Rueda-Tamayo Leidy ${ }^{1}$ \\ Almeida-Pérez Rafael ${ }^{2}$ \\ De la Rosa-Manjarrez Ginna² \\ Orozco-Gómez Fernando ${ }^{3}$ \\ Carrasquilla-Meléndez Rubén ${ }^{4}$ \\ Correspondencia: ruedatamayo@hotmail.com \\ Recibido para evaluación: abril - 15 - 2015. Aceptado para publicación: agosto - 20 - 2015
}

\section{RESUMEN}

Introducción: los aneurismas intracraneales son una dilatación local y permanente de una arteria.

Objetivo: describir las características clínicas, imagenológicas y la efectividad del tratamiento endovascular en términos de independencia y mortalidad en pacientes con aneurismas disecantes de circulación cerebral posterior en Cartagena.

Métodos: se realizó un estudio observacional descriptivo en pacientes de 18 años o mayores, con diagnósticos de aneurismas disecantes y de circulación cerebral posterior que recibieron tratamiento endovascular (TEV) en el período comprendido entre enero de 2007 y diciembre de 2014 en el centro Neurodinamia. Se midieron las variables relacionadas con antecedentes patológicos personales, diagnóstico y características del aneurisma, dispositivos utilizados para el tratamiento, tiempos de seguimiento clínico e imagenológico y complicaciones. Se realizó análisis estadístico descriptivo según la naturaleza de las variables.

Resultados: se analizaron 19 pacientes con tratamientos endovasculares. La mediana de edad fue de 55 años. La hemorragia subaracnoidea fue la presentación clínica más frecuente, el $89.5 \%$ de ellos fueron diagnosticados con TAC. La localización en la PICA y arteria vertebral fueron de $36.8 \%$ y $31.6 \%$, respectivamente. El tipo de dispositivos usados fueron los coils en $36.8 \%$, seguido de coils y stent con $31.6 \%$ y onyx en $21.0 \%$. Al finalizar la TEV se clasificó como resultado favorable en 18 pacientes $(94.7 \%)$, se presentó una muerte intrahospitalaria (5.3\%). La escala Rankin al seguimiento mostró mejoría en el $84.2 \%$ (16 pacientes).

\footnotetext{
Médico. Estudiante de Postgrado. Radiología. Facultad de Medicina. Universidad de Cartagena. Colombia. Médico. Especialista en Neurocirugía. Neurocirujano Endovascular. Neurodinamia. Cartagena. Colombia.

Médico. Neurorradiólogo. Intervencionista. Neurodinamia. Cartagena. Colombia.

Médico. Especialista en Anestesia y Reanimación. Neurodinamia. Cartagena. Colombia.
} 
ISSN: 2215-7840, 6(2), julio-diciembre 2015, Rueda-Tamayo Leidy, Almeida-Pérez Rafael, De la Rosa-Manjarrez Ginna, Orozco-Gómez Fernando, Carrasquilla-Melendez Ruben

Conclusiones: el TEV de los aneurismas disecantes de circulación cerebral posterior por oclusión del aneurisma muestra una alta frecuencia de éxito técnico $94.7 \%$, con un $84.2 \%$ de los pacientes con resultado favorable a los 30 días después del tratamiento. Rev.cienc.biomed. 2015;6(2):298-308

\section{PALABRAS CLAVE}

Aneurisma; Neurocirugía; Radiología intervencional.

\section{SUMMARY}

Introduction: the intracraneal aneurysms are local and permanent dilations in the artery.

Objective: to describe the clinical, imagenologic and effectiveness of endovascular treatment in terms of its independence and mortality in patients with dissecting aneurysms of posterior cerebral circulation in Cartagena.

Methods: a descriptive study was carried out with patients 18 years or older with a diagnosis of dissecting aneurysms of posterior cerebral circulation who received endovascular treatment in the period between January 2007 and December 2014 in the medical center Neurodinamia. The variables related to personal medical history, diagnosis and characteristics of the aneurysm, devices used for treatment, time clinical and imagenologic follow-up and complications were measured. Descriptive statistical analysis was performed according to the nature of the variables.

Results: nineteen patients were analyzed to receive endovascular treatments. The average of age was 55 years. The subarachnoid hemorrhage was the most frequent clinical case; this was diagnosed with CT in $89.5 \%$. The location in the PICA and vertebral artery were $36.8 \%$ and $31.6 \%$, respectively. The devices used were coils in $36.8 \%$, followed by coils and stent with $31.6 \%$, and Onyx with $21.0 \%$. At the end of VTE this was classified as favorable outcome in 18 patients $(94.7 \%)$; however, there was one death during hospital care $(5.3 \%)$. The Rankin scale showed improvement in $84.2 \%$ (16 patients).

Conclusions: the endovascular treatment of dissecting aneurysms posterior cerebral circulation and the aneurysm occlusion showed high success rates $94.7 \%$, patients $(84.2 \%)$ showed positive results after 30 days of treatment. Rev.cienc.biomed. 2015;6(2):298-308

\section{KEY WORDS}

Aneurysm; Neurosurgery; Radiology interventional.

\section{INTRODUCCIÓN}

Los aneurismas intracraneales son una dilatación local y permanente de una arteria. En la gran mayoría de los casos se trata de una lesión adquirida (1). La participación de factores ambientales como la hipertensión, el alcoholismo crónico y los pacientes fumadores activos, están bien documentados en el desarrollo de la lesión en la íntima de la pared arterial de los vasos intracraneales (2-4).

Existen tres tipos básicos de aneurismas: sacular, fusiforme y disecante (5). Los aneurismas disecantes se generan por un desgarro anormal en la íntima de la pared arterial, el cual produce una separación entre la íntima y la lámina elástica interna, provocando un hematoma intramural con la dilatación anormal de un lado de la pared arterial que, puede bloquear o reducir, el flujo sanguíneo a través de la arteria.
La forma de presentación va a variar de acuerdo a la localización de la disección dentro de la pared arterial, si ocurre entre la elástica interna y la media producirá un hematoma subintimal, el cual puede desencadenar la oclusión del vaso e isquemia. Si la disección ocurre entre la capa media y la adventicia, ocurrirá una hemorragia subaracnoidea (HSA), en estos casos con una elevada incidencia de resangrado y mortalidad (6).

Aunque los aneurismas disecantes son conocidos por inducir isquemia, recientes informes han demostrado un aumento de casos hemorrágicos (7). El estándar de oro para el diagnóstico es la angiografía con sustracción digital (DSA) y tiene como ventaja que también es utilizada en el abordaje terapéutico endovascular. Al ser un procedimiento invasivo se han reportado complicaciones neurológicas en un $0.5-1.8 \%$ y déficit permanente en $0.09-0.5 \%(8)$. 
La cirugía para los aneurismas disecantes de circulación posterior (ADCP) representa dificultades particulares como el recorte del cuello, debido a la profunda ubicación del aneurisma, y su estrecha relación con el tallo cerebral y los pares craneales. Las complicaciones postoperatorias de aneurismas de la arteria vertebral -PICA- reportadas incluyen disfagia, ronquera, diplopía, síndrome medular lateral y neumonía por aspiración. Los principales obstáculos de abordaje son el tubérculo yugular, cóndilo occipital y los pares craneales (9).

En la evaluación de la eficacia y la seguridad de las terapias es importante comparar el resultado del tratamiento esperado con la historia natural sin tratamiento. Los datos sobre la historia natural de los aneurismas disecantes de circulación posterior son limitados sin tratar. En general se piensa que la historia natural es desfavorable $(10,11)$.

La terapia endovascular (TEV) consiste en oclusión proximal con coils, oclusión del aneurisma con coils, con la asistencia de balón y/o stent, colocación de stent, o uso de líquido embolizante $(4,12,13)$. La TEV se ha establecido como terapia de primera línea en este tipo de aneurismas (4, 14).

En Colombia el manejo endovascular de los aneurismas cerebrales de circulación posterior se inició aproximadamente hace dos décadas, con la amplia aceptación de esta técnica. Sin embargo, pocos estudios se tienen sobre la efectividad de estas intervenciones. Solo existe una publicación de serie de casos sobre aneurismas de circulación posterior, en la cual 6 de 71 pacientes fueron disecantes (15).

Estas técnicas se han logrado implementar en Cartagena de Indias con un importante número de pacientes; sin embargo, aún no existe una descripción publicada de la efectividad de estas intervenciones. Cabe anotar que la disponibilidad de los servicios de terapia endovascular en Colombia son del $69 \%$, ligeramente inferior al de los países de Europa y América del Norte cuyo porcentaje es del $83 \%(16)$.

Hasta el momento no se cuenta con estudios prospectivos para definir la selección de los dispositivos más adecuados para la TEV. Se prefiere la oclusión proximal de la arteria madre después de HSA, en el estado agudo por ser técnicamente más fácil y en casos donde se involucra la PICA, puesto que permite una mejor circulación colateral, especialmente cuando las lesiones involucran el sitio de origen $(17,18)$. No obstante, se ha demostrado que no evita el resangrado (18, 19).

Esta investigación presenta la descripción demográfica y clínica de una serie consecutiva de pacientes, las características morfológicas de los aneurismas cerebrales presentes y asimismo, los resultados de la terapia endovascular y su seguimiento.

\section{MATERIALES Y MÉTODOS}

Se realizó un estudio observacional descriptivo tomando como población a todos los pacientes de ambos géneros, de 18 años o mayores con diagnósticos de aneurismas disecantes de circulación cerebral posterior que, recibieron tratamiento endovascular en el período comprendido entre enero de 2007 y diciembre de 2014 en el centro Neurodinamia, en las sedes Clínica Madre Bernarda y Nuevo Hospital Bocagrande de la ciudad de Cartagena.

La medición de variables relacionadas fue realizada con antecedentes patológicos personales que determinaron el estado de salud previo a la intervención, además, aspectos del diagnóstico del aneurisma y características del mismo. Se detallaron los dispositivos utilizados para la TEV como coils, stent, líquido embolizante, balones, y, finalmente, se anotaron los tiempos de seguimiento clínico e imagenológico y la aparición de complicaciones posteriores a la intervención endovascular, especialmente morbilidad y mortalidad postprocedimiento.

El análisis estadístico fue descriptivo y consistió en frecuencias absolutas y relativas en variables cuantitativas, medidas de tendencia central y dispersión en cuantitativas. La efectividad de la TEV fue reportada como la proporción de resultados favorables y ausencia de complicaciones y muerte postratamiento. 


\section{RESULTADOS}

En el período de los siete años de estudio se realizaron 19 tratamientos endovasculares para aneurismas disecantes de circulación cerebral posterior. El rango de edad de los pacientes estuvo entre 24 y 77 años con una mediana de 55 años (RI 47 - 59 años), el $53.6 \%$ de los casos se presentaron en mujeres (10 pacientes).

En el antecedente de hipertensión arterial se observó en $26.3 \%$ ( 5 pacientes). La clasificación de Hunt y Hess más frecuente de los aneurismas fue IV en $78.9 \%$ (15 pacientes), seguida de I con $15.8 \%$ (3 pacientes) y III $5.3 \%$ (1 paciente). Las presentaciones clínicas al momento del diagnóstico más frecuentes fueron la hemorragia subaracnoidea (HSA) con $84.2 \%$ y el hallazgo incidental con $10.5 \%$. La imagen diagnóstica más utilizada fue el TAC con $89.5 \%$ (ver Tabla No 1).

\begin{tabular}{|c|c|c|}
\hline \multicolumn{3}{|c|}{$\begin{array}{c}\text { TABLA No } 1 . \\
\text { CARACTERÍSTICAS GENERALES DE LOS } \\
\text { PACIENTES RELACIONADOS CON EL } \\
\text { DIAGNÓSTICO }\end{array}$} \\
\hline & $\mathrm{N}$ & $\%$ \\
\hline Edad Me [RI] & $55\left[\begin{array}{ll}47 & -59]\end{array}\right.$ & \\
\hline Sexo femenino & 10 & 53.6 \\
\hline Antecedente HTA & 5 & 26.3 \\
\hline \multicolumn{3}{|c|}{$\begin{array}{r}\text { Clasificación H\&H } \\
\end{array}$} \\
\hline I & 3 & 15.8 \\
\hline II & 0 & 0.0 \\
\hline III & 1 & 5.3 \\
\hline IV & 15 & 78.9 \\
\hline \multicolumn{3}{|c|}{ Presentación clínica } \\
\hline HSA & 16 & 84.2 \\
\hline Incidental & 2 & 10.5 \\
\hline Infarto embolia & 1 & 5.3 \\
\hline Efecto de masa & 0 & 0.0 \\
\hline \multicolumn{3}{|c|}{ Diagnóstico } \\
\hline TAC & 17 & 89.5 \\
\hline RM & 2 & 10.5 \\
\hline
\end{tabular}

Los hallazgos morfológicos más frecuentes de los aneurismas fueron la localización en la PICA y arteria vertebral en $36.8 \%$ y $31.6 \%$, respectivamente, tamaño pequeño de menos de $16 \mathrm{~mm}$ en $84.2 \%$, sin presencia de cuello o con cuello ancho en $42.1 \%$ y $31.6 \%$ (ver Tabla No 2).

\begin{tabular}{|l|c|c|}
\hline \multicolumn{3}{|c|}{ TABLA No 2.} \\
CARACTERÍSTICAS FÍSICAS DEL \\
ANEURISMA
\end{tabular}

El tiempo transcurrido entre el diagnóstico y el tratamiento endovascular fue menor de 24 horas en $89.5 \%$ ( 7 pacientes), el tipo de TEV fueron los coils en $36.8 \%$ (7 pacientes), seguido de coils+ stent $31.6 \%$ (6 pacientes) y onyx en $21.0 \%$ ( 4 pacientes). El uso de balón fue reportado en $42.1 \%$ (8 pacientes). Al finalizar la TEV se clasificó como resultado favorable en 18 pacientes (94.7\%), la complicación de muerte intrahospitalaria se presentó en la paciente con clasificación de resultado no favorable (5.3\%). De estos pacientes el $73.6 \%$ (en 14 pacientes se ocluyó el aneurisma y se preservó la circulación en el vaso madre) y en el $26.3 \%$ ( 5 pacientes) se ocluyó, totalmente, el vaso portador.

Los resultados de la escala Rankin al seguimiento mostraron mejoría en el $84.2 \%$ (16 pacientes), de los cuales el 68.4\% (13 pacientes) son pacientes asintomáticos, independientes o con síntomas menores $15.8 \%$ (3 pacientes), parcialmente dependientes y totalmente dependientes en $5.3 \%$ cada uno (un paciente en cada grado) (ver Tabla No $3)$. El seguimiento clínico se obtuvo en el $63.1 \%$ (12 pacientes). Se realizó seguimiento clínico mayor de un año en el $26.3 \%$ ( 5 pacientes), entre 1 y 3 meses $15.8 \%$ ( 3 pacientes), entre 4 y 6 meses o 10 y 12 meses con $10.5 \%$ (4 pacientes).

El seguimiento imagenológico estuvo disponible en $42.1 \%$ (8 pacientes), el intervalo 
de tiempo entre el tratamiento y el control imagenológico fue mayor de 1 año o de 4 a 6 meses con $15.8 \%$ cada uno ( 6 pacientes), 1 a 3 meses o de 10 a 12 meses con $5.3 \%$ cada uno (2 pacientes).

\begin{tabular}{|c|c|c|}
\hline \multicolumn{3}{|c|}{$\begin{array}{c}\text { TABLA NO } 3 . \\
\text { CARACTERÍSTICAS DEL TRATAMIENTO } \\
\text { ENDOVASCULAR Y RESULTADOS }\end{array}$} \\
\hline \multicolumn{3}{|c|}{ Tiempo entre el diagnóstico y el tratamiento } \\
\hline$<24$ horas & 17 & 89.5 \\
\hline 1 a 2 días & 0 & 0.0 \\
\hline 3 a 7 días & 1 & 5.3 \\
\hline 8 a 14 días & 1 & 5.3 \\
\hline \multicolumn{3}{|c|}{ Tipo de tratamiento endovascular } \\
\hline Coils & 7 & 36.8 \\
\hline Coils + stent & 6 & 31.6 \\
\hline Onyx & 4 & 21.0 \\
\hline Coils + onyx & 1 & 5.3 \\
\hline Stent & 1 & 5.3 \\
\hline Uso de balón & 8 & 42.1 \\
\hline \multicolumn{3}{|c|}{ Resultado de la intervención } \\
\hline Favorable & 18 & 94.7 \\
\hline No favorable & 1 & 5.3 \\
\hline \multicolumn{3}{|c|}{ Complicaciones } \\
\hline Resangrado & 1 & 5.3 \\
\hline Muerte hospitalaria & 1 & 5.3 \\
\hline \multicolumn{3}{|c|}{ Seguimiento } \\
\hline Escala de Ranking & & \\
\hline 0 Asintomático & 13 & 68.4 \\
\hline $\begin{array}{l}\text { I Independiente, síntomas } \\
\text { menores }\end{array}$ & 3 & 15.8 \\
\hline IV Parcialmente dependiente & 1 & 5.3 \\
\hline V Totalmente dependiente & 1 & 5.3 \\
\hline VI Muerto & 1 & 5.3 \\
\hline
\end{tabular}

\section{DISCUSIÓN}

Este es el primer estudio que describe los resultados del uso de terapia endovascular en aneurismas disecantes de circulación cerebral posterior en Colombia. Dentro de las ayudas diagnósticas de abordaje inicial en nuestros pacientes estuvo el TAC en el $85 \%$ de los casos, y en dos casos que consultaron ambulatoriamente, se utilizó resonancia magnética para el estudio de cefalea de origen vascular.

La primera opción de modalidad diagnóstica para los pacientes con sospecha de HSA aneurismática por su sensibilidad, disponibi- lidad y rapidez es la tomografía axial computarizada (TAC) sin contraste. La TAC detecta sangre en el espacio subaracnoideo en las primeras 12 horas con una sensibilidad de $98-100 \%$ y de $93 \%$ a las 24 horas.

Este rendimiento disminuye hasta $57-85 \%$ después de los primeros cinco días de inicio del cuadro clínico (nivel de evidencia 2 y grado de recomendación $B)(8,20)$. Con el TAC se puede observar el patrón de sangrado permitiendo sospechar un carácter aneurismático. Los patrones cortical puro y perimesencefálico son menos indicativos de aneurisma y de curso más benigno $(21,22)$. A todos los pacientes se les practicó confirmación diagnóstica con angiografía diagnóstica y sustracción (DSA), en la cual se describieron la morfología y el tamaño de los aneurismas.

No se ha evaluado sistemáticamente un posible vínculo de los aneurismas disecantes de circulación posterior con los factores de riesgo comunes para enfermedad vascular, tales como el consumo de tabaco, la hipertensión y el uso de anticonceptivos orales; un estudio caso-control sugirió la migraña como factor de riesgo (23). En el presente estudio se encontró una frecuencia más baja de antecedentes de hipertensión arterial (26.3\%), comparada con la frecuencia descrita en la literatura por Deng et al. 30\% y Kurata et al. $48 \%$, respectivamente $(21,24)$. Además se obtuvo un hallazgo incidental de anemia de células falciformes en nuestro paciente más joven (Figura No 1).

La incidencia global de disección de la arteria vertebral es de aproximadamente 1 a 1.5 por 100.000 casos de accidente cerebro vascular. Las disecciones espontáneas de la carótida y de la arteria vertebral son solo alrededor del $2 \%$ de todos los accidentes cerebrovasculares isquémicos, pero son una causa importante de ictus isquémico en pacientes jóvenes y de mediana edad y cuenta con el 10-25\% de estos casos (23). Aunque no es una patología tan frecuente como los síndromes coronarios agudos, su importancia radica en su elevada letalidad. Las disecciones espontáneas de las arterias vertebrales afectan a todos los grupos de edad, incluidos los niños, pero hay un pico 

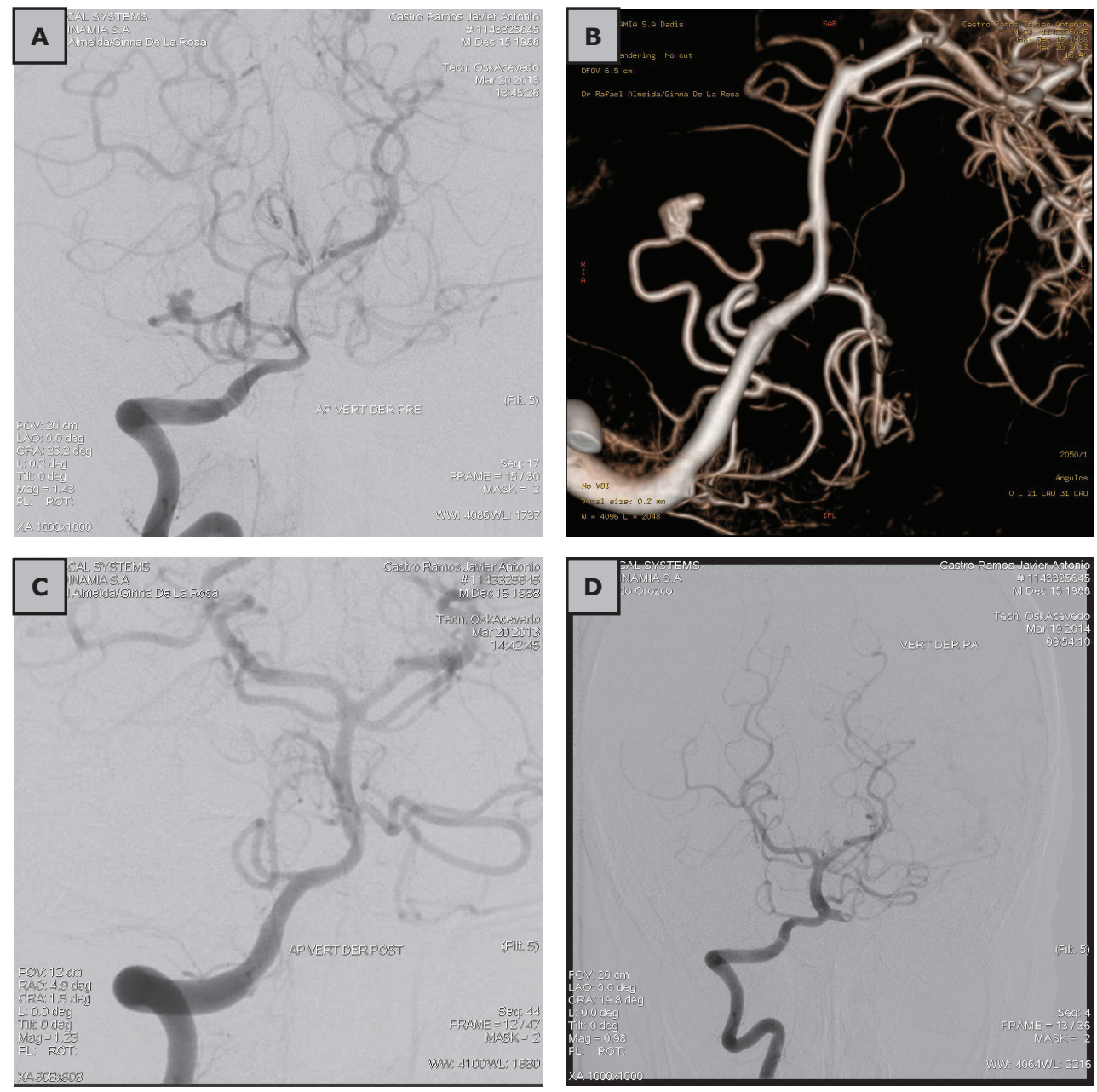

Figura No 1. Angiografías de paciente con terapia endovascular. A: DSA Anteroposterior de arteria vertebral derecha. Paciente de 24 años. HyH grado IV. Antecedente de anemia de células falciformes, HSA. Se aprecia aneurisma disecante de AICA (arteria cerebelosa anteroinferior); B: Reconstrucción 3D; C: DSA posterior a tratamiento. Obsérvese la oclusión completa de la arteria con líquido embolizante onyx; D: DSA seguimiento a 1 año. Paciente clínicamente independiente. Rankin 1. distinto en la quinta década de la vida (23), en este estudio tuvimos una media de edad de 53.9 años.

La tasa exacta de ocurrencia de aneurismas disecantes de la arteria vertebral con hemorragia subaracnoidea se desconoce, sin embargo, esta condición no es rara. Yamaura et al. (5) informó que $24(28 \%)$ de sus 86 pacientes con aneurismas de la arteria vertebral o sus ramas eran aneurismas disecantes, $21(87.5 \%)$ de estos pacientes se presentaron con HSA, y los otros tres (12.5\%), con isquemia (20). El porcentaje de aneurismas rotos en este estudio fue similar al de la literatura descrita.

En la serie descrita por Mizutani et al. (25), la ruptura temprana del aneurisma ocurrió antes del tratamiento en el $71 \%$ de los pacientes, y se manifestaron como HSA. El $69 \%$ de los 42 pacientes fueron sometidos a tratamiento. La mayoría de los 13 pacientes restantes que recibieron tratamiento conservador debido a la mala condición clínica o limitaciones anatómicas, murieron. Las rupturas de ADCP tienen una alta tasa de resangrado precoz (70\%).

El resangrado generalmente se produce dentro de las primeras 24 horas después de la ruptura inicial y se asocia con un pobre resultado clínico (7, 10-13, 17-19, 21-23, 26-28). En este estudio solo una paciente murió como resultado de la HSA crítica inicial. El $89.5 \%$ de los pacientes fueron tratados en las primeras 24 horas y no se detectó resangrado precoz en ninguno de estos, aunque los reportes de la literatura afirman que la oclusión proximal con coils parece no evitar el resangrado. No se presentó esta complicación.

En nuestro estudio, 15 pacientes (78.9\%) fueron clasificados en la escala de Hunt y 
Hess grado IV y se presentaron con coma transitorio y con recuperación entre 1 a 24 horas. Es un síntoma importante y característico de los ADCP; Mizutani et al. reportó que en 11 (26\%) de 42 pacientes (25) y Yamaura et al. en 7 (33\%) de 21 pacientes (5), con un porcentaje similar $8(33 \%)$ de 24 pacientes en el estudio de Kurata et al. (21).

En términos del tiempo de inicio de la terapia endovascular posterior al diagnóstico, más del $80 \%$ de los pacientes fueron tratados dentro de las 24 horas siguientes. Este resultado puede atribuirse a un amplio reconocimiento de la importancia de prevenir el resangrado ultratemprano y la disponibilidad del servicio endovascular.

En cuanto a la localización de la lesión y el modo del procedimiento, 7 pacientes (36.8\%) tenían compromiso de la PICA, 4 de estos fueron tratados con oclusión del aneurisma con coils, 1 con stent y coils, se demostró que la oclusión del aneurisma no afectó la relación de resultado favorable. Datos similares fueron obtenidos en el estudio JNET-II (20).

De acuerdo con el análisis de JNET-II, la obliteración completa de la lesión podría ser la preferida en el grupo que involucra la PICA como el papel más importante de terapia endovascular para aneurismas vertebrales disecantes rotos, y prevención de la rotura recurrente. Endo et al. (19) mostró que el infarto medular postoperatorio se asocia con resultados desfavorables después de la oclusión del aneurisma con coils y de los ADCP rotos. En esta serie se preservó la PICA en 6 pacientes, los cuales no presentaron complicaciones.

En lo relacionado con las publicaciones de los resultados de tratamiento endovascular, los estudios disponibles son retrospectivos; el porcentaje de resultado favorable es variable desde el 51 hasta el 95\%. Esto va a depender de la presentación clínica, roto o no roto; la escala de Hunt y Hess al ingreso o de la World Federation of Neurologic Surgeons (WFNS), de la pericia de los médicos tratantes y de los dispositivos utilizados (9, $13,18,20,21,29,30)$.
Hasta el momento no se cuenta con estudios prospectivos para definir la selección de los dispositivos más adecuados para la TEV. Se prefiere la oclusión proximal del vaso madre después de HSA, en el estado agudo por ser técnicamente más fácil y en casos donde se involucra la PICA porque se puede permitir una mejor circulación colateral. Especialmente cuando las lesiones involucran el sitio de origen $(17,18)$; sin embargo, se ha considerado que no evita el resangrado $(18,19)$.

En la técnica con oclusión del aneurisma con coils, donde se inserta el primer coil, proximal a la disección. Luego con un nuevo catéter de inserción distal se inserta un coil distal, y con él se evita manipular la disección (23). Actualmente existen variaciones a este tipo de oclusiones (20). Puede ser más eficaz que la oclusión proximal para aneurismas disecantes vertebrales rotos debido al cese inmediato del flujo de sangre de los sitios afectados (21).

Es tendencia que la técnica de elección sea la oclusión del aneurisma con coils, anteriormente descrita $(18,19)$. En nuestro estudio se realizó oclusión del aneurisma con preservación del vaso madre en $73.6 \%$ (14 pacientes), de los cuales el $36.8 \%$ (7 pacientes) fueron intervenidos solo con coils.

La introducción de los stents en el tratamiento endovascular permitió la preservación de la arteria madre afectada. Esta técnica "reconstructiva" se sugirió para alterar la dinámica de flujos intra-aneurismáticos y conducir a una formación de trombo dentro del aneurisma $(22,28)$. Se indicó en qué casos los aneurismas son fusiformes o de cuello ancho. Actualmente se utilizan con técnica combinada en el uso de coils $(9,13,18,20)$. El stent único es eficaz y seguro en aneurismas donde no es considerada la oclusión proximal de la arteria madre, al parecer múltiples stent superpuestos son más eficaces que un solo stent (27).

El uso de stent parece ser viable en el caso de preservar la PICA (26). Existen complicaciones tromboembólicas frecuentes y necesidad de terapia antiplaquetaria a largo plazo (31). Con el desarrollo de los stent diver- 
ISSN: 2215-7840, 6(2), julio-diciembre 2015, Rueda-Tamayo Leidy, Almeida-Pérez Rafael, De la Rosa-Manjarrez Ginna,

Orozco-Gómez Fernando, Carrasquilla-Melendez Ruben

sores de flujo, la superficie de metal denso $(30 \%-35 \%)$ y buena navegabilidad, están presentes en el mismo dispositivo. Estos stents pueden modificar la hemodinámica aneurismática, y producir trombosis intraaneurismática, previniendo así, la HSA recurrente.

Presentan baja porosidad con celdas muy pequeñas, completamente diferente de los stents intracraneales utilizados como soporte para la embolización con coils, en los que las celdas son bastante grandes para facilitar la cateterización aneurismática. Además, los stent diversores de flujo inducen crecimiento de las células endoteliales, proporcionando un estrechamiento del cuello y una disminución del flujo en el interior del aneurisma $(32,33)$.

Narata en su reporte de dos casos utilizó diversores de flujo, informó que estos pueden ser una alternativa para el tratamiento de aneurismas disecantes de arteria vertebral dominante o cuando la PICA está involucrada. La disposición multicapa de los stent diversores de flujo pueden proporcionar oclusión del aneurisma, con la preservación de la arteria y la remodelación de la ramas (34).

El $31.8 \%$ (6 pacientes) fueron tratados con coils más stent y el $5.3 \%$ (1 paciente) fue tratado solo con stent, cuatro pacientes que tenían compromiso de la arteria vertebral, 1 de la arteria basilar y 1 de la PICA. Solo en un caso de stent y coil se presentó recanalización, no se evidenciaron complicaciones tromboembolias.

El líquido embolizante onyx es un agente no-adhesivo que está compuesto por copolímeros de etileno y alcohol vinílico disuelto en sulfóxido de dimetilo. Está aprobado para embolización prequirúrgica de malformaciones arteriovenosas cerebrales (Onyx 18 y 34 ) y para el tratamiento de los aneurismas en la pared lateral con cuello ancho (ONYX HD-500), pero se ha utilizado para numerosas aplicaciones fuera de las recomendadas. El onyx (cianoacrilato de n-butilo) se ha utilizado para el sacrificio vaso principal en tratamientos de aneurisma disecantes, y micóticos cerebrales periféricos (35).
Estos tratamientos pueden ser complicados por pequeños infartos que son generalmente asintomáticos o mínimamente sintomáticos. Por otra parte, la oclusión terapéutica de un vaso proximal tal como la carótida interna - la arteria vertebral son típicamente realizados con coils, ya que los agentes líquidos plantean el riesgo de un accidente cerebrovascular embólico significativo.

Existen pocos estudios prospectivos con utilización de onyx en aneurismas de circulación posterior, entre ellos: CAMEO-2004, Piske et al. y Simon et al. 2014 (36-38), en los cuales el número de aneurismas disecantes tratados han sido 19 casos en total. Existen pocos datos en la literatura para soportar una conclusión definitiva de si es más peligroso el uso de líquido embolizante en la circulación cerebral posterior o en la anterior.

El $21 \%$ (4 pacientes) fueron tratados con líquido embolizante onyx, de estos 1 caso con compromiso de la PICA y 3 con compromiso de la arteria cerebelosa anteroinferior (AICA). Todos con un resultado favorable. Dentro de la revisión de la literatura de los estudios prospectivos disponibles no se halló descripción del compromiso de la AICA. Mahmoud et al. (39) en su reporte de tres casos describe que los aneurismas disecantes de la AICA corresponden al 1\% de todos los aneurismas cerebrales y la oclusión de la arteria madre con líquido embolizante es factible y eficiente en términos de la prevención de resangrado. Las complicaciones neurológicas postembolización son impredecibles, y dependen del lugar de la oclusión y la presencia de las arterias colaterales cerebelosas, lo cual concuerda con los resultados aquí mostrados.

En esta serie falleció una paciente con HSA grado IV, con aneurisma clasificado como grande, sin cuello y que comprometía la arteria vertebral, para su tratamiento se usó coils y más líquido embolizante; no obstante, la paciente falleció en el primer día del postoperatorio. El resultado se consideró desfavorable a su estado clínico inicial.

En cuanto al uso del balón, su objetivo fue remodelar el vaso antes de la colocación de coils o stent. En los reportes actuales, su ne- 
cesidad sigue siendo un enigma. Los méritos de este método son la prevención de embolia distal y la reducción del sangrado de la hemorragia subaracnoidea en el momento de ruptura en el intraprocedimiento. Se utilizó balón en el 42\% ( 8 pacientes) de los procedimientos en esta serie, cuatro de los pacientes tenían compromiso de la PICA, no documentaban complicaciones tromboembólicas, cosa que contrasta con lo reportado por Satow et al. (20).

Kurata et al. reportó eventos tromboembólicos en uno de sus 18 pacientes, quien presentaba aneurisma disecante de arteria vertebral rota, con infarto en territorio PICA (21). No hubo complicaciones perioperatorias en 14 pacientes tratados ya sea con colocación de stent o stent con coils en la serie de $A H N$, en este último estudio seis de los 12 pacientes, en el seguimiento angiográfico a los 6-12 meses, mostraron oclusión incompleta (40).

En el estudio de Lihara, la morbilidad relacionada con el tratamiento fue de $16.7 \%$, y de los 18 pacientes, 12 no tuvieron la participación de PICA y seis pacientes si tuvieron tal compromiso (41). Rabinov et al. informó un resultado favorable en el $68 \%$ de los 25 pacientes tratados con $20 \%$ de mortalidad en 3.5 años promedio de seguimiento clínico (30). Buena recuperación fue reportada en el $64 \%$ de los pacientes que se presentaron con HSA y $100 \%$ presentó buena recuperación en los pacientes con aneurismas distales de la arteria vertebral por Lihara y colegas (41). Deng et al. reportó una evolución excelente en 37 de sus pacientes y una considerada buena (24). Este análisis sugiere que el tratamiento endovascular de los aneurismas VA es factible, con una aceptable baja morbilidad y mortalidad perioperatoria. Los resultados son consistentes con las observaciones anteriores.

La recanalización se observó en dos pacientes que tenían compromiso de la arteria vertebral, los aneurismas fueron clasificados como grandes, uno con cuello favorable que fue tratado con coils y uno sin cuello que fue tratado con stent y coils. Se les realizó un nuevo tratamiento. Los datos publicados hasta el momento sobre la recurrencia des- pués del tratamiento se refieren principalmente a los aneurismas saculares, y pocos estudios se han centrado en la recurrencia de aneurismas disecantes de arteria vertebral (42). El período de tiempo transcurrido fue de 3 y 6 meses respectivamente. Se hizo hincapié en la necesidad de seguimiento a largo plazo con estudios angiográficos. El retratamiento puede ser necesario en caso de recanalización o recrecimiento del pseudoaneurisma (24).

Al respecto Zhao et al. en su estudio de cohorte de 94 pacientes indicó que el efecto de la interacción entre la implantación del stent, la participación de la PICA, y el grado de oclusión inmediata están estrechamente asociados con la recurrencia después del tratamiento reconstructivo de $\operatorname{ADCP}(42)$.

Este estudio presentó limitaciones propias de su diseño descriptivo: en primer lugar, el tamaño de la muestra es pequeño dada la ocurrencia de los aneurismas de estudio; segundo, el seguimiento clínico y angiográfico fue realizado por el médico tratante. Dicho esto, se requieren estudios analíticos que controlen mejor la confusión y permitan comparar de manera más objetiva los diferentes tipos de intervenciones.

\section{CONCLUSIÓN}

El tratamiento endovascular de los aneurismas disecantes de circulación cerebral posterior, por oclusión del aneurisma con coils, stent y coils y líquido embolizante onyx muestran una gran tasa de éxito técnico hasta del $94.7 \%$, con un $68.4 \%$ de los pacientes con resultado favorable a los 30 días después del tratamiento. Los resultados de este estudio se pueden usar como datos de referencia para estudios futuros en nuestro país.

CONFLICTOS DE INTERESES: ninguno que declarar.

FINANCIACIÓN: recursos propios de los autores. Honorarios profesionales, costos clínico-hospitalarios e insumos fueron cubiertos dentro de la atención médico asistencial. 


\section{REFERENCIAS BIBLIOGRÁFICAS}

1. Yamaura I, Tani E, Yokota M, Nakano A, Fukami M, Kaba K, et al. Endovascular treatment of ruptured dissecting aneurysms aimed at occlusion of the dissected site by using Guglielmi detachable coils. Journal of neurosurgery. 1999;90(5):853-6.

2. Bassi P, Bandera R, Loiero M, Tognoni G, Mangoni A. Warning signs in subarachnoid hemorrhage: a cooperative study. Acta neurologica scandinavica. 1991;84(4):277-81.

3. Fontanarosa PB. Recognition of subarachnoid hemorrhage. Annals of emergency medicine. $1989 ; 18(11): 1199-205$.

4. Molyneux AJ, Kerr RS, Yu L-M, Clarke M, Sneade M, Yarnold JA, et al. International subarachnoid aneurysm trial (ISAT) of neurosurgical clipping versus endovascular coiling in 2143 patients with ruptured intracranial aneurysms: a randomised comparison of effects on survival, dependency, seizures, rebleeding, subgroups, and aneurysm occlusion. The Lancet. 2005;366(9488):809-17.

5. Yamaura A, Watanabe $Y$, Saeki N. Dissecting aneurysms of the intracranial vertebral artery. Journal of neurosurgery. 1990;72(2):183-8.

6. Krings T, Choi I. The many faces of intracranial arterial dissections. Interventional Neuroradiology. 2010;16(2):151.

7. Andoh T, Shirakami S, Nakashima T, Nishimura Y, Sakai N, Yamada H, et al. Clinical analysis of a series of vertebral aneurysm cases. Neurosurgery. 1992;31(6):987-93.

8. Bederson JB, Connolly ES, Batjer HH, Dacey RG, Dion JE, Diringer MN, et al. Guidelines for the management of aneurysmal subarachnoid hemorrhage a statement for healthcare professionals from a special Writing Group of the Stroke Council, American Heart Association. Stroke. 2009;40(3):994-1025.

9. Lv X, Jiang C, Li Y, Wu Z. Clinical outcomes of ruptured and unruptured vertebral artery-posterior inferior cerebellar artery complex dissecting aneurysms after endovascular embolization. American Journal of Neuroradiology. 2010;31(7):1232-5.

10. Bacigaluppi $S$, Piccinelli $M$, Antiga $L$, Veneziani $A$, Passerini $T$, Rampini $P$, et al. Factors affecting formation and rupture of intracranial saccular aneurysms. Neurosurgical review. 2014;37(1):1-14.

11. Liaw N, Fox JMD, Siddiqui AH, Meng H, Kolega J. Endothelial nitric oxide synthase and superoxide mediate hemodynamic initiation of intracranial aneurysms. PloS one. $2014 ; 9(7):$ e101721.

12. Brown Jr RD, Broderick JP. Unruptured intracranial aneurysms: epidemiology, natural history, management options, and familial screening. The Lancet Neurology. 2014;13(4):393-404.

13. Pandey AS, Koebbe C, Rosenwasser RH, Veznedaroglu E. Endovascular Coil Embolization of Ruptured and Unruptured Posterior Circulation Aneurysms: Review of A 10 Year Experience. Neurosurgery. 2007;60(4):626-37.

14. Group P. Determinants of poor outcome after aneurysmal subarachnoid hemorrhage when both clipping and coiling are available: Prospective Registry of Subarachnoid Aneurysms Treatment (PRESAT) in Japan. World neurosurgery. 2011;76(5):437-45.

15. Ascencio JL, Vargas SA, Jiménez CM, Arias SA. Terapia endovascular en aneurismas vertebrobasilares: experiencia del grupo neurovascular (junio de 1996-octubre de 2004). Rev colomb radiol. 2006;17(3):1986-94.

16. Alcalá-Cerra G, Paternina JJG, de Ávila MEB, Mesa EIP, Barrios RS, Niño-Hernández LM, et al. Treatment of aneurysmal subarachnoid hemorrhage and unruptured intracranial aneurysms by neurosurgeons in Colombia: A survey. Surgical neurology international. $2011 ; 2$.

17. Jin S-C, Kwon D, Choi C-G, Ahn J, Kwun B-D. Endovascular strategies for vertebrobasilar dissecting aneurysms. American Journal of Neuroradiology. 2009;30(8):1518-23.

18. Sugiu K, Tokunaga K, Watanabe K, Sasahara W, Ono S, Tamiya T, et al. Emergent endovascular treatment of ruptured vertebral artery dissecting aneurysms. Neuroradiology. 2005;47(2):15864.

19. Endo H, Matsumoto $Y$, Kondo R, Sato K, Fujimura M, Inoue T, et al. Medullary infarction as a poor prognostic factor after internal coil trapping of a ruptured vertebral artery dissection: Clinical article. Journal of neurosurgery. 2013;118(1):131-9.

20. SaTow T, Ishii D, Iihara K, Sakai N. Endovascular Treatment for Ruptured Vertebral Artery Dissecting Aneurysms: Results from Japanese Registry of Neuroendovascular Therapy (JR-NET) 1 and 2. Neurologia medico-chirurgica. 2014;54(2):98-106.

21. Kurata A, Ohmomo T, Miyasaka Y, Fujii K, Kan S, Kitahara T. Coil embolization for the treatment of ruptured dissecting vertebral aneurysms. American Journal of Neuroradiology. 2001;22(1):118.

22. Lv X, Jiang C, Li Y, Wu Z. Clinical outcomes of lower cranial nerve palsies caused by vertebral artery-posteroinferior cerebellar artery aneurysms after endovascular embolization. Neurological research. 2010;32(8):796-800.

23. Park K-W, Park J-S, Hwang S-C, Im S-B, Shin W-H, Kim B-T. Vertebral artery dissection: natural history, clinical features and therapeutic considerations. Journal of Korean Neurosurgical Society. 2008;44(3):109-15.

24. Deng D, Jin D, Zhou J, Chang Q, Qu K. Characteristics and endovascular treatment of intracranial vertebral artery aneurysms. Neurology India. 2011;59(6):833. 
25. Mizutani T, Aruga T, Kirino T, Miki Y, Saito I, Tsuchida T. Recurrent subarachnoid hemorrhage from untreated ruptured vertebrobasilar dissecting aneurysms. Neurosurgery. 1995;36(5):905-13.

26. Kim M, Chung J, Kim S, Roh H, Kwon B, Kim B-S, et al. Stenting from the vertebral artery to the posterior inferior cerebellar artery. American Journal of Neuroradiology. 2012;33(2):34852.

27. Park S, Kim B, Kim D, Shin Y, Suh S, Chung E, et al. Clinical and angiographic follow-up of stent-only therapy for acute intracranial vertebrobasilar dissecting aneurysms. American Journal of Neuroradiology. 2009;30(7):1351-6.

28. Wakhloo AK, Lanzino G, Lieber BB, Hopkins LN. Stents for intracranial aneurysms: the beginning of a new endovascular era? Neurosurgery. 1998;43(2):377-9.

29. Edlow JA, Fisher J. Diagnosis of Subarachnoid Hemorrhage Time to Change the Guidelines? Stroke. 2012;43(8):2031-2.

30. Rabinov JD, Hellinger FR, Morris PP, Ogilvy CS, Putman CM. Endovascular management of vertebrobasilar dissecting aneurysms. American Journal of Neuroradiology. 2003;24(7):1421-8.

31. Rho MH, Park HJ, Chung EC, Choi YJ, Lee SY, Won YS, et al. Various techniques of stentassisted coil embolization of wide-necked or fusiform artherosclerotic and dissecting unruptured vertebrobasilar artery aneurysms for reducing recanalization: mid-term results. Acta neurochirurgica. 2013;155(11):2009-17.

32. Lylyk P, Miranda C, Ceratto R, Ferrario A, Scrivano E, Luna HR, et al. Curative endovascular reconstruction of cerebral aneurysms with the pipeline embolization device: the Buenos Aires experience. Neurosurgery. 2009;64(4):632-43.

33. Kulcsár Z, Wetzel SG, Augsburger L, Gruber A, Wanke I, Rüfenacht DA. Effect of flow diversion treatment on very small ruptured aneurysms. Neurosurgery. 2010;67(3):789-93.

34. Narata AP, Yilmaz H, Schaller K, Lovblad KO, Pereira VM. Flow-diverting stent for ruptured intracranial dissecting aneurysm of vertebral artery. Neurosurgery. 2012;70(4):982-9.

35. Oh DC, Hirsch JA, Yoo AJ. Novel use of onyx for treatment of intracranial vertebral artery dissection. Journal of neurointerventional surgery. 2012;4(1):31-3.

36. Molyneux AJ, Cekirge S, Saatci I, Gál G. Cerebral Aneurysm Multicenter European Onyx (CAMEO) trial: results of a prospective observational study in 20 European centers. American Journal of Neuroradiology. 2004;25(1):39-51.

37. Piske RL, Kanashiro LH, Paschoal E, Agner C, Lima SS, Aguiar PH. Evaluation of onyx HD500 embolic system in the treatment of 84 wide-neck intracranial aneurysms. Neurosurgery. 2009;64(5):E865-E75.

38. Simon S, Archer K, Mericle R. Multicenter registry of liquid embolic treatment of cerebral aneurysms. World neurosurgery. 2014;82(6):e731-e8.

39. Mahmoud M, El Serwi A, Habib MA, Gamrah SA. Endovascular Treatment of AICA Flow Dependent Aneurysms: A Report of Three Cases and Review of the Literature. Interventional Neuroradiology. 2012;18(4):449.

40. Ahn J, Han I, Kim T, Yoon P, Lee Y, Lee B-H, et al. Endovascular treatment of intracranial vertebral artery dissections with stent placement or stent-assisted coiling. American Journal of Neuroradiology. 2006;27(7):1514-20.

41. Iihara K, Sakai N, Murao K, Sakai H, Higashi T, Kogure S, et al. Dissecting aneurysms of the vertebral artery: a management strategy. Journal of neurosurgery. 2002;97(2):259-67.

42. Zhao K-J, Zhao R, Huang Q-H, Xu Y, Hong B, Fang Y-B, et al. The Interaction between Stent(s) Implantation, PICA Involvement, and Immediate Occlusion Degree Affect Symptomatic Intracranial Spontaneous Vertebral Artery Dissection Aneurysm (sis-VADA) Recurrence after Reconstructive Treatment with Stent(s)-Assisted Coiling. Euro Radiol. 2014;24(9):2088 $-96$. BIOMÉDICAS
Es el órgano de información científica de la Facultad de Medicina de la Universidad de Cartagena. Colombia. Publique su trabajo en esta revista enviando su manuscrito a: revistacienciasbiomedicas@unicartagena.edu.co www.revistacienciasbiomedicas.com www.revistacienciasbiomedicas.com.co

Revista ciencias Biomédicas es una publicación independiente, imparcial, abierta, revisada por pares, de elevada visibilidad internacional, con circulación online e impresa. Publica artículos en todas las modalidades universalmente aceptadas en inglés y en español, de temas referentes con todas las ciencias biomédicas, incluyendo ámbitos clínicos, epidemiológicos o de estudios básicos.

El sistema de gestión de manuscritos es rápido y justo. Revista Ciencias Biomédicas está incluida en varias bases de datos latinoamericanas e internacionales.

Antes de enviar su manuscrito, revise las recomendaciones para los autores, presentes en: www.revistacienciasbiomedicas.com www.revistacienciasbiomedicas.com.co 